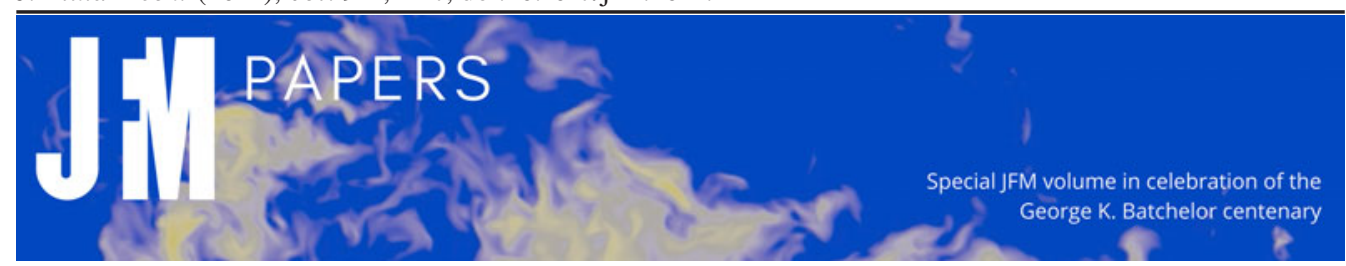

\title{
Scalings of the mixing velocity for buoyancy-driven instabilities in porous media
}

\author{
S.S. Gopalakrishnan ${ }^{1,2, \dagger, \text { B. Knaepen }}{ }^{2}$ and A. De $\mathrm{Wit}^{1, \dagger}$ \\ ${ }^{1}$ Nonlinear Physical Chemistry Unit, Université libre de Bruxelles (ULB), CP231, 1050 Brussels, Belgium \\ ${ }^{2}$ Université libre de Bruxelles (ULB), Physique des Systèmes Dynamiques, CP231, \\ 1050 Brussels, Belgium
}

(Received 4 June 2020; revised 4 November 2020; accepted 10 January 2021)

A miscible horizontal interface separating two solutions of different solutes in the gravity field can deform into convective finger structures due to the Rayleigh-Taylor (RT) instability, or the double-diffusive (DD) and diffusive-layer-convection (DLC) instabilities, triggered by differential diffusion of the solutes. We analyse here numerically the nonlinear dynamics of these buoyancy-driven instabilities in porous medium flows by an integration of Darcy's law coupled to advection-diffusion equations for the concentrations of the two solutes. After a diffusive growth, the mixing length $L$, defined as the vertical extent of the mixing zone, starts to grow linearly when convection sets in. We compute the mixing velocity $U$ as the slope of this linear growth. In the one-species RT regime, $U$ is proportional to $\Delta \rho_{0}$, the initial density difference between the two layers. In the two-species problem, differential diffusion effects can induce non-monotonic density profiles characterised by an adverse density difference, defined as the density jump across the spatial domain where the density decreases along the direction of gravity. We find that, in the parameter space spanned by the buoyancy ratio $R$, and the ratio $\delta$ of diffusion coefficients of the two species, the mixing velocity scales linearly with this dynamic density difference. It is computed analytically from the diffusive base-state density profile and can be significantly larger than $\Delta \rho_{0}$. Our results evidence the possibility of controlling the mixing of RT, DD and DLC instabilities in two-species stratifications by a careful choice of the nature and thus diffusivity of the species involved.

Key words: buoyancy-driven instability, double diffusive convection, convection in porous media

$\dagger$ Email addresses for correspondence: shyam7sunder@gmail.com, adewit@ulb.ac.be 


\section{S.S. Gopalakrishnan, B. Knaepen and A. De Wit}

\section{Introduction}

Buoyancy-driven flows are among the most common flows encountered in nature as well as in engineering applications. G. K. Batchelor already long ago pointed to the genericity of free convection, i.e. convective motions due to buoyancy forces, among the fundamental problems in fluid dynamics (Batchelor 1954). We analyse here buoyancy-driven instabilities for porous medium flows, focusing on the case of two-species stratifications. Free convection deformation of an initially horizontal miscible interface separating two solutions in a porous medium occurs in a variety of contexts. Examples range from carbon dioxide sequestration (Huppert \& Neufeld 2014; De Wit 2016) to geological flows (Scott \& Stevenson 1986), reaction-driven flows (Almarcha et al. 2010; De Wit 2020), ground water sedimentation (Menand \& Woods 2005), refining techniques (Hill 1952) as well as biological flows (Dullien 2012) to name a few. In these scenarios, the convective motion arises as a result of different instability mechanisms.

The Rayleigh-Taylor (RT) instability develops at the interface when a denser solution overlies a less dense one in the gravity field, deforming it into finger-like structures (Manickam \& Homsy 1995; Fernandez et al. 2001; Martin, Rakotomalala \& Salin 2002; Trevelyan, Almarcha \& De Wit 2011; Gopalakrishnan et al. 2017; De Paoli, Zonta \& Soldati 2019). In the case of one-species stratifications, the RT instability develops when a solution of a given solute overlies a less concentrated solution of the same solute. For porous medium flows, the first experimental studies carried out by Hill (1952) and Wooding (1969) considered the stratification of a sugar or salt aqueous solution above water in a Hele-Shaw cell (two glass plates separated by a thin gap) (Batchelor 1967). In the presence of an unstable stratification (denser on top of less dense), the interface was seen to rapidly deform into fingers. A mixing zone starts to develop, which is defined as the region where the two miscible fluids mix and the density, averaged along the transverse direction, departs from that of the two fluids. After a transient diffusive growth, the mean vertical amplitude of the mixing zone enters a nonlinear regime in which it grows linearly in time, while the mean wavelength of the fingers increases as $t^{1 / 2}$. Several numerical (Jenny et al. 2014; Slim 2014; Gopalakrishnan et al. 2017; De Paoli et al. 2019) or experimental works either in Hele-Shaw cells (Fernandez et al. 2002; Menand \& Woods 2005) or real three-dimensional porous media (Nakanishi et al. 2016; Teng et al. 2017) have shown that the vertical mixing zone between the two solutions scales proportionally to the initial density difference $\Delta \rho_{0}$ between the two solutions.

When two different solutes are involved, differential diffusion effects can also destabilise an initially statically stable stratification (less dense on top of denser) if the two solutes making opposing contributions to the vertical density gradient have different molecular diffusivities (Huppert \& Turner 1981). The densities of the layers are controlled by the concentrations of each solute while their nature fixes the ratio of diffusivities. Two cases can be encountered depending on whether the fast diffusing species is dissolved in the upper or the lower layer.

When the solute of the denser lower solution diffuses faster than the solute present in the upper less dense layer, a double-diffusive (DD) instability can induce a fingered deformation of the interface (Turner 1979; Green 1984; Huppert \& Sparks 1984; Cooper, Glass \& Tyler 1997; Pringle \& Glass 2002; Trevelyan et al. 2011; Radko 2013). Such a DD instability has been extensively studied in the context of salt fingers that form in oceans where thermohaline convection is triggered by the differential diffusion of salt and heat (Schmitt 1994; Schmitt et al. 2005). DD fingering has been analysed experimentally in Hele-Shaw cells by Pringle \& Glass (2002) starting from a less dense sucrose solution overlying a denser salt solution. At a fixed buoyancy ratio, they observed that the vertical 


\section{Scaling of buoyancy-driven fingers in porous media}

finger length scales linearly with time. Although a scaling law was not investigated in their work, their data show that the velocity with which the fingers move vertically remains almost the same, even when concentrations are varied, provided the ratio between these concentrations is kept constant.

When the solute in the upper less dense solution is the one that diffuses faster, a so-called diffusive-layer-convection (DLC) instability can arise (Turner \& Stommel 1964; Turner 1979; Griffiths 1981; Huppert \& Turner 1981; Trevelyan et al. 2011). It is due to the fact that, when the upper solute diffuses faster downwards, it creates a depletion zone above the initial contact line while it accumulates below the interface. Locally unstable density stratifications, i.e. zones where the density decreases along the direction of gravity, develop then on either side of the interface. They drive locally convective motions that propagate independently through the solutions. The DLC mechanism, which features convection rolls on either side of the interface, was investigated experimentally by Stamp et al. (1998) using solutions of salt above sucrose. Scaling laws for the convective velocities have been obtained as a function of the buoyancy flux.

The RT, DD and DLC instabilities have been shown recently to arise genuinely in stratifications of reactive solutions as they involve different solutes with different diffusion coefficients (Lemaigre et al. 2013; De Wit 2020). This has motivated us to revisit the scalings of the RT instability in two-species stratifications of non-reactive fluids, as well as its possible interaction with the DD and DLC differential diffusion modes (Trevelyan et al. 2011; Carballido-Landeira et al. 2013; Donev et al. 2015; Gopalakrishnan et al. 2018).

In that spirit, the interplay between RT and DLC modes has been analysed for initially unstable interfaces of a denser solution of a fast-diffusing solute above a less dense solution of a slow-diffusing solute (Carballido-Landeira et al. 2013; Donev et al. 2015; Gopalakrishnan et al. 2018). An RT-DLC 'mixed-mode' regime has been identified with a deformation of the miscible interface having features from both RT and DLC instabilities. The interface deforms in a sinusoidal shape thanks to the RT mode. The fact that the upper solute diffuses faster downwards induces, however, also a depletion zone above and an accumulation below the caps of the RT deformation. As a result, local convective rolls, signature of the DLC mode, deform the head of the RT fingers into 'Y-shaped' antennae at the location of the local DLC-driven adverse density gradients (Carballido-Landeira et al. 2013).

Similarly, the influence of DD modes on the RT instability has been recently investigated both experimentally and numerically (Gopalakrishnan et al. 2018). Because of DD effects, the RT density profiles are non-monotonic and feature a dynamic density difference $\Delta \rho_{m}$ which is larger than the unstable initial density stratification $\Delta \rho_{0}$ because of the faster upwards diffusion of the solute in the lower layer. In the nonlinear regime, the mixing length (defined as the distance between the top and bottom of the fingered zone) grows linearly with time. The slope of this linear growth defines the mixing velocity $U$ characterising how fast the two solutions mix. It was found that the mixing velocity $U$ scales with $\Delta \rho_{m}$ calculated from the base flow configuration. The scaling law measured experimentally was in excellent agreement with the one obtained in numerical simulations. Of particular interest is that this dynamic density difference $\Delta \rho_{m}$ can be computed analytically from the base-state density profiles when the two species in the stratification are known.

These results confirm the fact that non-monotonic density profiles can trigger new effects on the RT instability, as shown also in stratified RT turbulence (Lawrie \& Dalziel 2011; Davies Wykes \& Dalziel 2014), and for porous medium flows with density profiles that are a linear combination of step profiles (Gandhi \& Trevelyan 2014). In particular, 


\section{S.S. Gopalakrishnan, B. Knaepen and A. De Wit}

analysis of the RT-DD regime shows that two-species stratifications feature interesting new dynamics and scalings (Gopalakrishnan et al. 2018) but a large part of the parameter space remains unaddressed. In particular, it is of interest to analyse whether DLC modes can have a similar impact on RT scalings. In addition, as pure DD and DLC regimes also feature in some cases non-monotonic density profiles, the question arises to what extent the specificities of these profiles control the mixing velocity.

In this context, the aim of the present study is to obtain the scaling laws governing the mixing velocity of buoyancy-driven fingering dynamics for two-species stratification in porous media. We explore numerically the scalings of the mixing velocity for porous medium flows in the whole parameter space spanned by the buoyancy ratio $R$, which is a measure of the initial density difference between the two solutions, and the ratio $\delta$ of diffusion coefficients of the two species. Specifically, we extend previously obtained results for the RT-DD flows (Gopalakrishnan et al. 2018) to the regime of RT-DLC interplay, and to the pure DD and DLC regimes. We find that, in all regimes, the mixing velocity scales linearly with the adverse dynamic density difference which can simply be computed analytically using the values of $R$ and $\delta$. These results enlighten some previously obtained experimental results (Pringle \& Glass 2002).

To this end, after introducing the geometry of the system and the governing equations in $\S 2$, the base-state density profiles and flow features in the $(R, \delta)$ parameter space are presented in $\S 3$. The scaling laws obtained for RT flows influenced by DD modes (Gopalakrishnan et al. 2018) are summarised in $\S 4$. The findings are extended to the regimes where only DD effects are present in $\S 5$. The scenarios where RT effects are coupled with the DLC mechanism are investigated in $\S 6$. The transition to the RT-DLC mixed-mode regime, and eventually to the case where only DLC is present, complements the study. The paper finishes with a summary and a comparison with previous experiments in $\S 7$ before giving the main conclusions in $\S 8$.

\section{Geometry and governing equations}

Let us consider two different miscible solutions that are in contact along a horizontal interface in a two-dimensional porous medium or a Hele-Shaw cell with gravity pointing downwards. The horizontal interface is chosen as the $x$-axis while the $y$-axis is the vertical direction increasing downwards. The upper solution contains a solute A while the lower solution contains a solute $\mathrm{B}$, with initial concentrations $A_{0}$ and $B_{0}$ respectively. The solutions are assumed to be dilute such that the diffusion coefficients $D_{A}$ and $D_{B}$ of species $\mathrm{A}$ and $\mathrm{B}$ respectively can be assumed constant. The density is taken to vary linearly with the concentrations as $\rho(A, B)=\rho_{0}\left[1+\alpha_{A} A+\alpha_{B} B\right]$, where $\rho_{0}$ is the density of the solvent, $A$ and $B$ are the concentration of the respective species and $\alpha_{A}, \alpha_{B}$ are the solutal expansion coefficients defined as $\alpha_{A}=\left(1 / \rho_{0}\right)(\partial \rho / \partial A)$ and $\alpha_{B}=\left(1 / \rho_{0}\right)(\partial \rho / \partial B)$. The flow dynamics around this miscible interface is described by Darcy's equation coupled to advection-diffusion equations for the concentrations $A$ and $B$. The set of equations governing the system are non-dimensionalised by the characteristic velocity $\mathcal{U}=g K \alpha_{A} A_{0} / \mu$, length $\mathcal{L}=D_{A} / \mathcal{U}$ and time $\mathcal{T}=\mathcal{L} / \mathcal{U}$, where $g$ is the magnitude of the acceleration due to gravity, $K$ is the permeability and $\mu$ is the dynamic viscosity. The concentrations are non-dimensionalised using $A_{0}$ while the density is scaled as $\left(\rho / \rho_{0}-1\right) / \alpha_{A} A_{0}$. The resulting non-dimensional equations read (Trevelyan et al. 2011; Gopalakrishnan et al. 2018)

$$
\begin{gathered}
\nabla p=-\boldsymbol{u}+(A+R B) \hat{\boldsymbol{y}}, \\
\nabla \cdot \boldsymbol{u}=0,
\end{gathered}
$$


Scaling of buoyancy-driven fingers in porous media

$$
\begin{aligned}
& A_{t}+\boldsymbol{u} \cdot \nabla A=\nabla^{2} A, \\
& B_{t}+\boldsymbol{u} \cdot \nabla B=\delta \nabla^{2} B,
\end{aligned}
$$

where $p$ is the pressure, $\boldsymbol{u}$ is the Darcy flow velocity, $\hat{\boldsymbol{y}}$ is the unit vector in the direction of gravity, $R=\alpha_{B} B_{0} / \alpha_{A} A_{0}$ the dimensionless buoyancy ratio and $\delta=D_{B} / D_{A}$ the ratio of the diffusion coefficients. Note that the buoyancy ratio $R$ can be seen as the ratio of the Rayleigh number of the lower species $B$ with respect to that of the upper species $A$. The system is closed by the following initial conditions:

$$
\left.\begin{array}{ll}
A=1, B=0, \boldsymbol{u}=0 & \text { for } y<0, \\
A=0, B=1, \boldsymbol{u}=0 & \text { for } y>0 .
\end{array}\right\}
$$

The boundary conditions are $A=1, B=0, \boldsymbol{u}=0$ for $y \rightarrow-\infty$, and $A=0, B=1, \boldsymbol{u}=0$ for $y \rightarrow \infty$.

The nonlinear dynamics is analysed by numerical simulations performed using the finite-volume code YALES2 (Moureau, Domingo \& Vervisch 2011; Gopalakrishnan et al. 2017, 2018). The simulations were run in a large square box domain $(102400 \times 102400$ non-dimensional units) with periodic boundary conditions along the horizontal direction, while we impose no flux for the concentrations of the species in the vertical direction and vanishing normal velocity $\left(\boldsymbol{u}_{\boldsymbol{n}}=0\right)$ on the upper and lower walls. This box is large enough for the dynamics not to be influenced by the upper and lower boundaries, with around 100 fingers developing across the interface and simulations running for up to $5 \times 10^{6}$ time units. The instability is triggered by adding a small amount of noise with an amplitude of $0.1 \%$ on the initial concentration fields (2.5) throughout the entire domain. The numerical averages presented in this work represent an average over 20 simulations with each simulation starting with a different random noise. Typical fingering patterns of the density fields for RT, DD, and DLC scenarios are shown in figure 1 and are discussed in more detail in the subsequent sections.

\section{Base-state density profiles and flow features}

In the absence of convection, the dimensionless base-state concentration profiles are analytical solutions of the diffusion equations (2.3) and (2.4) with $\boldsymbol{u}=\mathbf{0}$. Assuming a domain of infinite height, they are given by

$$
\bar{A}(y, t)=\frac{1}{2} \operatorname{erfc}\left(\frac{y}{2 \sqrt{t}}\right), \quad \bar{B}(y, t)=\frac{1}{2} \operatorname{erfc}\left(-\frac{y}{2 \sqrt{\delta t}}\right) .
$$

The corresponding non-dimensional base-state density profile can be constructed as

$$
\bar{\rho}(y, t)=\bar{A}(y, t)+R \bar{B}(y, t) .
$$

At $t=0$, when the initial dimensionless concentration profiles follow a step function, the density in the upper layer $\bar{\rho}_{A}=1$ as $\bar{A}=1$ and $\bar{B}=0$. In the lower layer where $\bar{A}=0$ and $\bar{B}=1, \bar{\rho}_{B}=R$. The dimensionless initial density jump $\Delta \rho_{0}$ between the two layers, defined as the difference between the initial upper and lower densities, is thus $\Delta \rho_{0}=$ $\bar{\rho}_{A}-\bar{\rho}_{B}=1-R$. It is fixed by the buoyancy ratio $R$ and is independent of the diffusivity ratio $\delta$ as the two species have not started mixing by diffusion yet. Note that, as $\bar{\rho}(y, t)+$ $\bar{\rho}(-y, t)=1+R$, the convective patterns in buoyancy-driven flows evolve the same way on both sides of the initial interface (Trevelyan et al. 2011). 


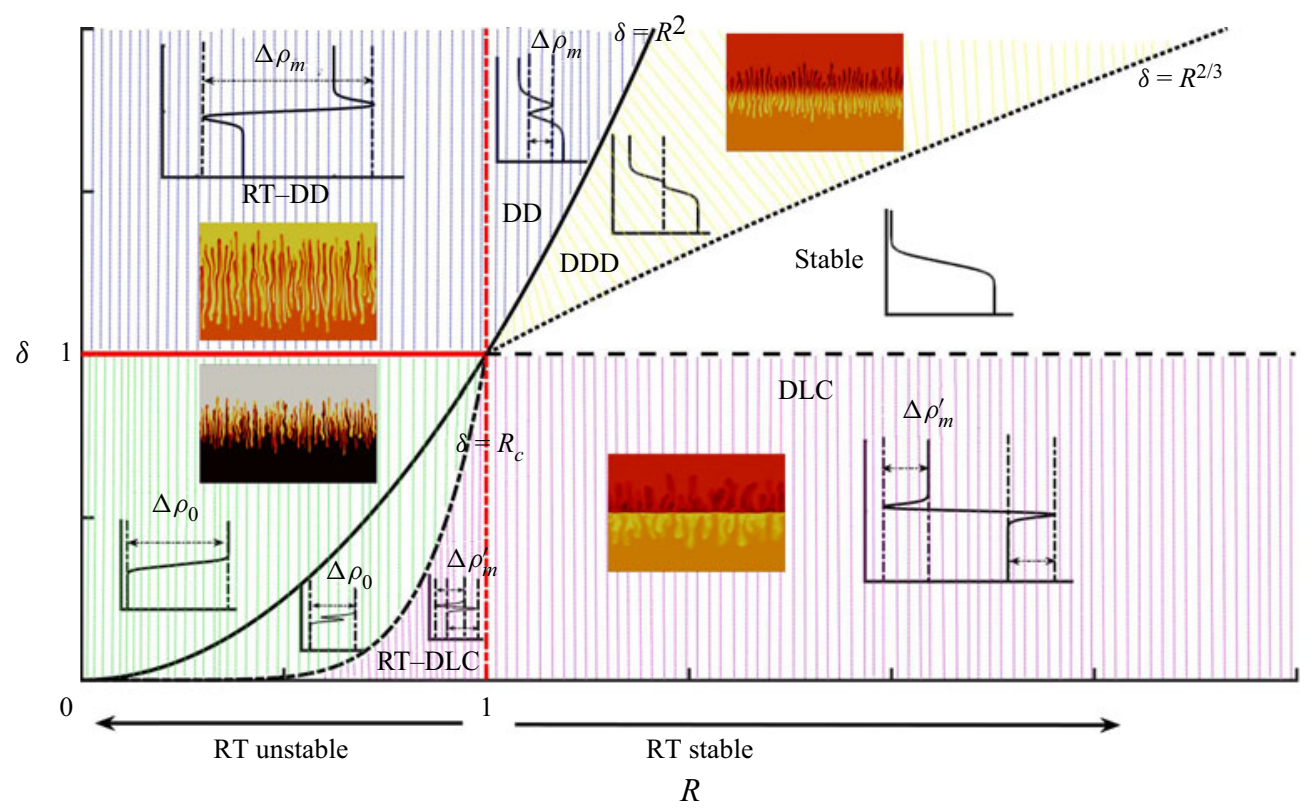

Figure 1. Typical analytical diffusive base-state density profiles $\bar{\rho}(y, t)$ in the $(R, \delta)$ parameter space. Representative spatial distributions of the density field in the nonlinear regime illustrate the fingering patterns. The shaded blue region corresponds to the parameter space where the RT instability is influenced by DD effects (RT-DD regime for $R<1, \delta>1)$ and where pure DD develops $\left(R>1\right.$ and $\left.1<R^{2}<\delta\right)$. The green region is the zone where the RT instability dominates for $R_{c}<\delta<1$. The pink region corresponds to the RT-DLC mixed-mode regime $\left(\delta<R_{C}<1\right)$, and the pure DLC zone $(R>1, \delta<1)$. The yellow and white regions correspond to base-state density profiles which are monotonically increasing $\left(R>1,1 \leq \delta \leq R^{2}\right)$. The mixing velocity $U$ scales as $U=0.8 \Delta \rho_{m}$ (blue region), $U=0.8 \Delta \rho_{0}$ (green region) and $U=0.8 \Delta \rho_{m}^{*}=0.4 \Delta \rho_{m}^{\prime}$ (pink region).

For $R<1$, we have a RT unstable configuration (denser above less dense) with an interface that deforms into fingers developing similarly above and below the interface. In particular, the one-species RT instability occurs when $R<1$ and $\delta=1$ denoted by the red full line in figure 1. The flow dynamics is self-similar in $R$ on this line $\delta=1$. The stratifications with $R>1, \delta=1$ are stable. At later times, different dynamic density stratifications can be obtained depending on the values of parameters $R$ and $\delta$.

When $\delta>1$, the upward diffusion of the lower solute B is faster than the downward diffusion of the upper solute A. In the RT regime $(R<1)$, the density profiles develop then a non-monotonic spatial dependence as soon as $t>0$ with a maximum above the initial interface and a minimum in the lower layer (Trevelyan et al. 2011; Gopalakrishnan et al. 2018). As shown in figure 1, this induces a dynamic density difference $\Delta \rho_{m}>0$ which is larger than the initial density difference $\Delta \rho_{0}$ and controls the vertical speed of the RT fingers (Gopalakrishnan et al. 2018). Section 4 summarises the influence of $\Delta \rho_{m}$ on the flow dynamics and scalings in this RT regime influenced by DD effects (called here the RT-DD regime). When $R \geq 1$, the less dense solution is initially overlying the denser one but, when $\delta>1$, the faster diffusion of solute B to the upper layer can induce a DD instability. The asymptotic neutral stability curve is given by $\delta=R^{2 / 3}$ (thick dotted line in figure 1) below which the stratification is linearly stable (Trevelyan et al. 2011; Gopalakrishnan 2020). For $R^{2 / 3} \leq \delta \leq R^{2}$, the miscible interface is initially statically stable but deforms at a later time via a delayed-double-diffusive (DDD) instability 
(Trevelyan et al. 2011). The DDD flows are not investigated in the present study as we have considered only flows that are readily unstable at $t=0$. Moreover, as shown in figure 1 , the density profiles are monotonically increasing downwards when $1 \leq \delta \leq R^{2}$ (shaded yellow and white regions in figure 1), and they feature thus no unstable stratification neither globally nor locally. We will focus here on the DD zone $\left(1 \leq R^{2} \leq \delta\right.$ when $\left.R>1\right)$ for which non-monotonic profiles are obtained. Scalings in this pure DD regime are discussed in $\S 5$.

If $\delta<1$, the solute A present in the upper phase diffuses faster downwards than the solute $\mathrm{B}$ which diffuses upwards. If $R \geq 1$, the initial density stratification is stable but, as soon as $t>0$, the differential diffusion between solutes induces the formation of a depletion zone above the initial contact line and an accumulation region below it. This triggers a so-called DLC instability because the density profiles in these regimes are non-monotonic with two local zones possessing an unstable stratification where the density decreases along the direction of gravity (Trevelyan et al. 2011; Carballido-Landeira et al. 2013). Localised convection develops then on either side of the miscible interface. If $R<1$, the properties of the RT regime depend on the value of $\delta$. For $R^{2}<\delta<1$, the density profiles are monotonically decreasing downwards (figure 1) and classical RT dynamics is observed with fingers deforming in a similar manner above and below the initial interface. If $\delta<R^{2}$, a non-monotonic spatial dependence is observed in the density profiles with a locally stable density stratification across the initial miscible interface as a result of the faster downward diffusion of the solute in the upper layer. A critical value for the buoyancy ratio, $R_{c}$, can be defined for which the unstable initial density difference, $\Delta \rho_{0}$, equals the density difference $\Delta \rho_{m}$ across the locally stable zone around the initial interface (Carballido-Landeira et al. 2013). This boundary corresponds to the $\delta=R_{c}$ curve in figure 1 which is given by $\delta=R^{n}$ (where $n \approx 6.8$ ) (Carballido-Landeira et al. 2013). It has been shown that, for $R_{c}<\delta<R^{2}$, i.e. when the initial unstable density jump, $\Delta \rho_{0}$, exceeds the amplitude $\Delta \rho_{m}$ of the locally stable zone, RT effects dominate in the deformation of the miscible interface. However, the presence of the locally stable zone due to the differential diffusion effects deforms the cap of the fingers in a 'mushroom-like' way (Carballido-Landeira et al. 2013). When $\delta<R_{c}<1$, the local stable stratification across the interface has a larger density jump than the initial unstable density difference, giving an RT-DLC mixed-mode regime in which RT flow features are influenced by DLC effects (Carballido-Landeira et al. 2013) (called here RT-DLC regime). A 'Y-shaped antenna-like' structure of the tip of the fingers is then observed on either side of the interface, which is itself deformed by the RT modulation. At a fixed $\delta<1$, Carballido-Landeira et al. (2013) showed that the contribution of the RT mode decreases while the DLC characteristics become more and more prominent when $R$ increases, in agreement with the numerical simulations of Trevelyan et al. (2011). Typical base-state density profiles in these regimes are illustrated in figure 1. Scalings in the RT-DLC, and pure DLC regimes are discussed in $\S 6$. A more complete description of the various features of the base-state density profiles can be found in Trevelyan et al. (2011).

\section{Scaling in the RT-DD regime $(R<1, \delta>1)$}

The scalings in the $R<1, \delta>1$ zone of the parameter space i.e. in the RT regime influenced by DD effects have been analysed previously by Gopalakrishnan et al. (2018) but are summarised in this section for completeness. At $t=0$, we start from a statically unstable density stratification characterised by the initial density difference $\Delta \rho_{0}=1-R$ (shown in red in figure $2 a$ ). As $\delta<1$, the solute $\mathrm{B}$ in the lower less dense solution diffuses faster upwards than the solute $\mathrm{A}$ in the upper denser zone which diffuses downwards. 


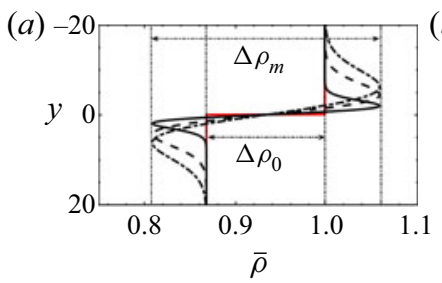

(d) 1.10

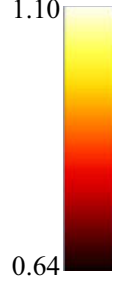

(b)

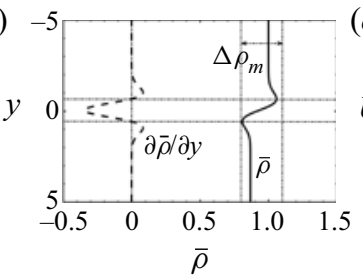

(c)

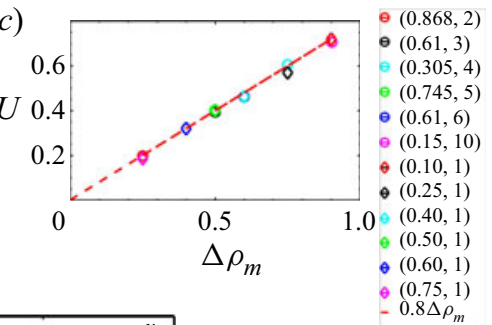

$0.8 \Delta \rho_{m}$

(e)
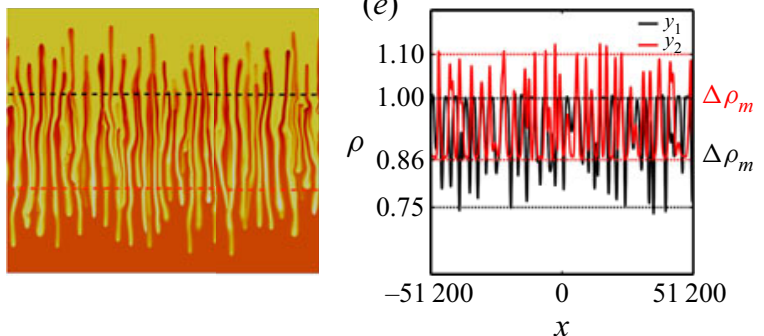

Figure 2. Characteristics of the RT-DD regime when DD modes influence the RT instability $(R<1, \delta>1)$. Here, $R=0.868, \delta=2$. (a) Self-similar base-state density profiles at time $t=0,1,5,10$. The initial profile at $t=0$, shown in red, features the initial density jump, $\Delta \rho_{0}$ whereas, as soon as $t>0$, a dynamic density jump $\Delta \rho_{m}$ is triggered by the differential diffusion effects. (b) Base-state density profile $\bar{\rho}(y)$ (solid curve) and its derivative $\partial \bar{\rho} / \partial y$ (dotted curve). (c) Mixing velocity $U$ as a function of the dynamic density jump $\Delta \rho_{m}$ (values of $R, \delta$ are shown for each colour point). The red line is the scaling $U=0.8 \Delta \rho_{m}$. The maximum deviation in the values of $U$ arising from the seeded initial conditions over 20 simulations is of the order $10^{-3}$. (d) Spatial distribution of the density at a given time in the nonlinear regime. The density profiles along the black and red lines denoted in $(d)$ are shown in $(e)$ with the amplitude of $\Delta \rho_{m}, 0.25$, demarcated using dotted lines and marked as labels along the $y$ axis.

This leads to non-monotonic base-state density profiles with a maximum in the upper layer and a minimum in the lower one as soon as $t>0$ (figure $2 a$ ). As a result, a dynamic density jump $\Delta \rho_{m}>0$ is obtained across the zone where $\partial \bar{\rho} / \partial y<0$ and that corresponds to the density difference between the maximum and the minimum. This adverse density jump $\Delta \rho_{m}$ is larger than the initial density difference $\Delta \rho_{0}$, stays constant for $0<t<\infty$, and can be computed analytically as (Gopalakrishnan et al. 2018)

$$
\Delta \rho_{m}=\operatorname{erf}\left(\sqrt{\frac{\ln \left|\left(\delta / R^{2}\right)\right|}{2|(1-1 / \delta)|}}\right)+R \operatorname{erf}\left(-\sqrt{\frac{\ln \left|\left(\delta / R^{2}\right)\right|}{2|(\delta-1)|}}\right) .
$$

As an example, the base-state density profile for $R=0.868, \delta=2$ and its derivative along the vertical direction are shown in figures $2(a)$ and $2(b)$ respectively. Note that, if $\delta=1$ in (4.1), we recover $\Delta \rho_{m}=\Delta \rho_{0}=1-R$.

It has been shown recently both experimentally and numerically that, for $\delta>1$, this adverse dynamic density jump $\Delta \rho_{m}$ (rather than $\Delta \rho_{0}$ ) governs the velocity $U$ at which the mixing zone of the RT fingers grows (Gopalakrishnan et al. 2018). This mixing velocity $U$ is computed as the slope in the nonlinear regime of the temporal evolution of the averaged mixing length $L$ measured as the distance between the most upward and downward point of the fingered zone. As shown in figure 2(c), $U$ scales as $U=0.8 \Delta \rho_{m}$ in the zone of parameter space where $R<1$ and $\delta \geq 1$ (Gopalakrishnan et al. 2018).

This scaling of $U$ can be qualitatively understood from the spatial distribution of the density field in the nonlinear regime, as shown in figure $2(d)$. The variations of the density along the black and red lines passing in the middle of the upper and lower parts respectively 


\section{Scaling of buoyancy-driven fingers in porous media}

of the fingered zone are shown in figure 2(e). The density difference between the middle of the fingers and the invaded solution ahead of it is seen to be approximately equal to $\Delta \rho_{m}$ $(=0.25)$. This remains the same during the whole nonlinear regime. This shows that, even though the initial density jump is $\Delta \rho_{0}$, right from the beginning, differential diffusion induces a non-monotonic density profile such that, locally, fingers experience the dynamic density difference $\Delta \rho_{m}$ with the solution surrounding them. As a consequence, the mixing velocity $U$ at which the fingers move in their environment remains constant and scales as $\Delta \rho_{m}$ (Gopalakrishnan et al. 2018). It is worth noting that the value of $\Delta \rho_{m}$ is independent of the flow equation and can be computed analytically via (4.1) solely on the basis of the solution to the diffusive equations for the concentrations of the two solutes at hand. In the subsequent sections, we extend the scaling observed for the RT-DD regime to other regimes in the $(R, \delta)$ parameter space.

\section{Scaling in the pure DD regime $\left(1 \leq R^{2} \leq \delta\right)$}

The pure DD instability arises when $1 \leq R^{2} \leq \delta$ starting from an initially statically stable configuration (less dense above denser). A couple of representative base-state density profiles are shown for these cases in figure $3(a, b)$. The initial density profile (shown in red in figure $3 a, b)$ is buoyantly stable $\left(\Delta \rho_{0}=(1-R)<0\right)$. However, as $\delta>1$, B diffuses faster upwards than A, which diffuses downwards. As soon as $t>0$, this induces a non-monotonic density profile with, locally, a denser zone above a less dense one across the initial interface. The amplitude of this buoyantly unstable density difference is the dynamic density jump $\Delta \rho_{m}>0$, which can be computed using the values of $(R, \delta)$ via (4.1). This region with an adverse density gradient is the zone where $\partial \bar{\rho} / \partial y<0$, as shown for the corresponding base-state profiles in figure $3(c, d)$.

We have numerically integrated model (2.1)-(2.4) for various values of $R$ and $\delta$ in the pure DD regime. The spatial distribution of the density field at a given time in the nonlinear regime is shown in figure $4(a, c)$ for two values of parameters in the $R \geq 1, \delta \geq 1$ zone. As both RT and DD instabilities have similar eigenfunctions (Trevelyan et al. 2011), similar convective patterns are observed in the case of pure DD. Figure 4 shows some examples of DD fingers in the spatio-temporal distribution of the density field. From such snapshots, we can measure the mixing length as the distance between the upper and lower points of the mixing zone. Figure 5(a) shows the temporal evolution of the mixing length $L$ averaged over 20 simulations for various values of $\delta>1$ at $R=1.1$. We see that a linear scaling can be observed in the nonlinear regime, such as in the RT regime (Gopalakrishnan et al. 2017; De Paoli et al. 2019). The mixing velocity $U$, computed as the slope of this linear $L(t)$ trend, is shown as a function of $\delta$ for three different values of $R$ in figure $5(b)$. At a fixed $R$, it increases with $\delta$, while for a given value of $\delta$ it decreases when $R$ increases. As observed in the RT-DD regime, the mixing velocity scales here linearly again with the dynamic density jump as $U=0.8 \Delta \rho_{m}$ (figure $5 c$ ). To understand this scaling, we plot in figure $4(b, d)$ the variation of density along the black and red lines shown in figures $4(a)$ and $4(c)$ respectively. Strikingly, the adverse density jump $\Delta \rho_{m}$ induced by differential diffusion effects is the density difference between the middle of the fingers and their surroundings. This explains why their averaged propagation speed quantified by $U$ scales with $\Delta \rho_{m}$.

In the DDD regime obtained in the parameter space for $R^{2 / 3} \leq \delta \leq R^{2}$, the first derivative of the base-state density profiles is strictly positive throughout the domain. Typical density profiles along with their spatial derivatives are illustrated for this regime in figure 6. Although the flows are linearly unstable, the differential diffusion effects do not lead to locally unstable zones where $\partial \bar{\rho} / \partial y<0$ in the base-state density profiles. 
(a)

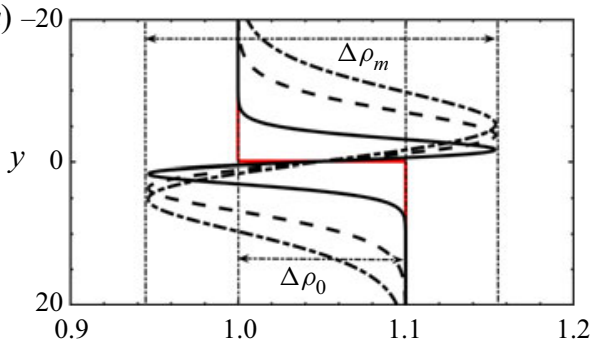

(c)

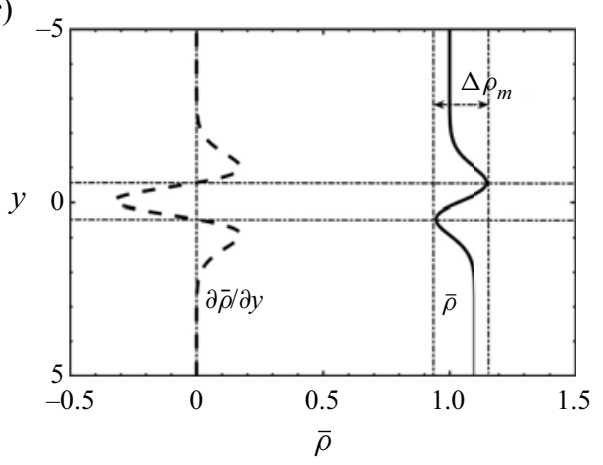

(b) -20

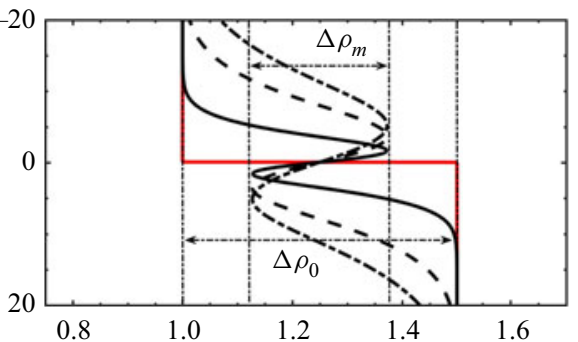

(d)

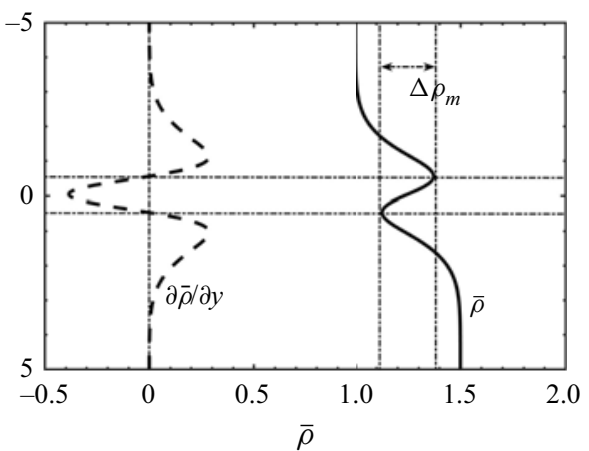

Figure 3. Characteristics of the pure DD regime $\left(1 \leq R^{2} \leq \delta\right)$. $(a, b)$ Base-state density profiles shown at $t=0$ (in red) and at 3 successive times for $(a) R=1.1, \delta=3$ and $(b) R=1.5, \delta=7$. $(c, d)$ Base-state density profile $\bar{\rho}(y)$ and its derivative $\partial \bar{\rho} / \partial y$ for the parameter settings in $(a, b)$. The dynamic density jump $\Delta \rho_{m}$ that governs the rate at which the fingers grow corresponds to the difference in density between the bounds of the interval where $\partial \bar{\rho} / \partial y$ is negative.

(a)

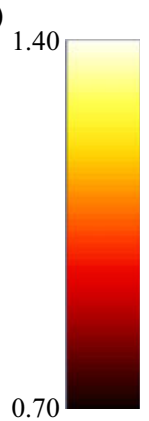

(c)

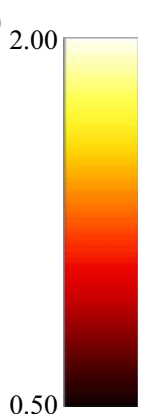

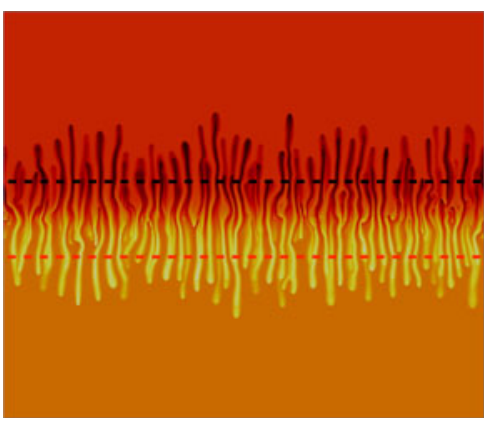

(b)

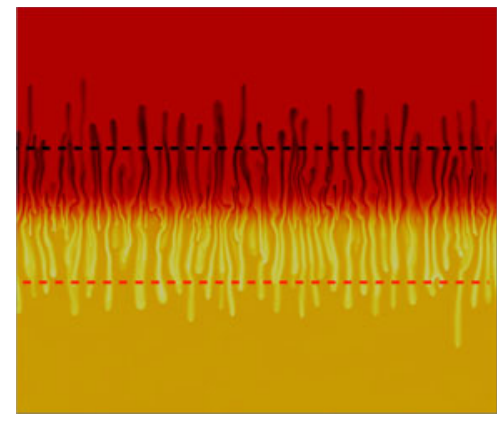

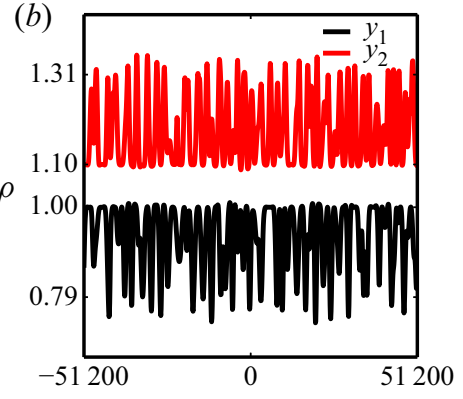

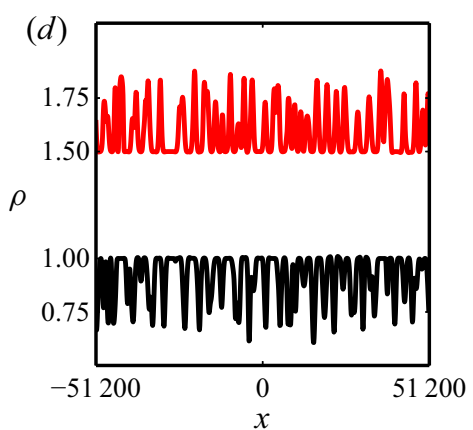

Figure 4. Spatial distribution of the density field at a given time in the nonlinear regime for $(a) R=1.1, \delta=3$ and $(a, c) R=1.5, \delta=7$. The variations of the density along the black and red lines in $(a, c)$ are shown in $(b, d)$. The corresponding values of $\Delta \rho_{m}$ are 0.21 and 0.25 respectively. 

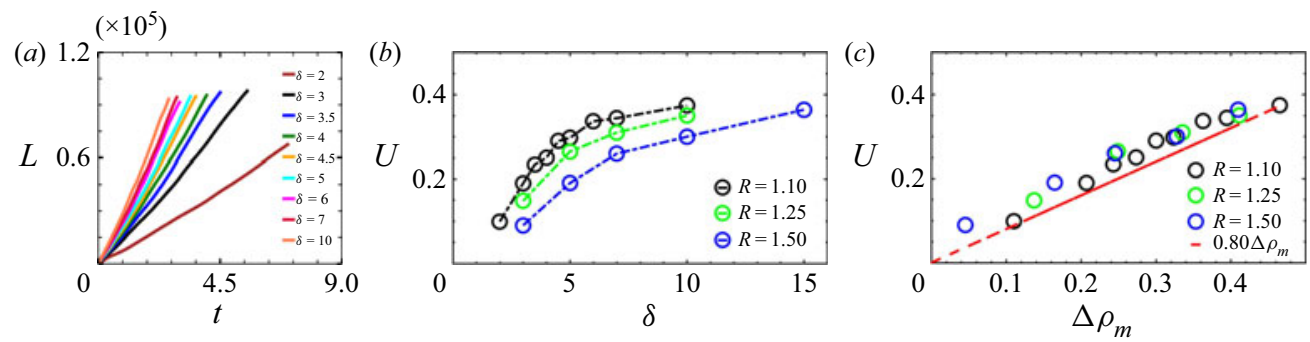

Figure 5. (a) Temporal evolution of the mixing length $L$ (averaged over 20 simulations) for $R=1.1$ and various values of $\delta$. Mixing velocity $U$ as a function of $(b) \delta$ and $(c) \Delta \rho_{m}$ at three different values of $R$. The red line in $(c)$ is the scaling $U=0.8 \Delta \rho_{m}$. The maximum deviation in the values of $U$ arising from the seeded initial conditions over 20 simulations is of the order $10^{-3}$.
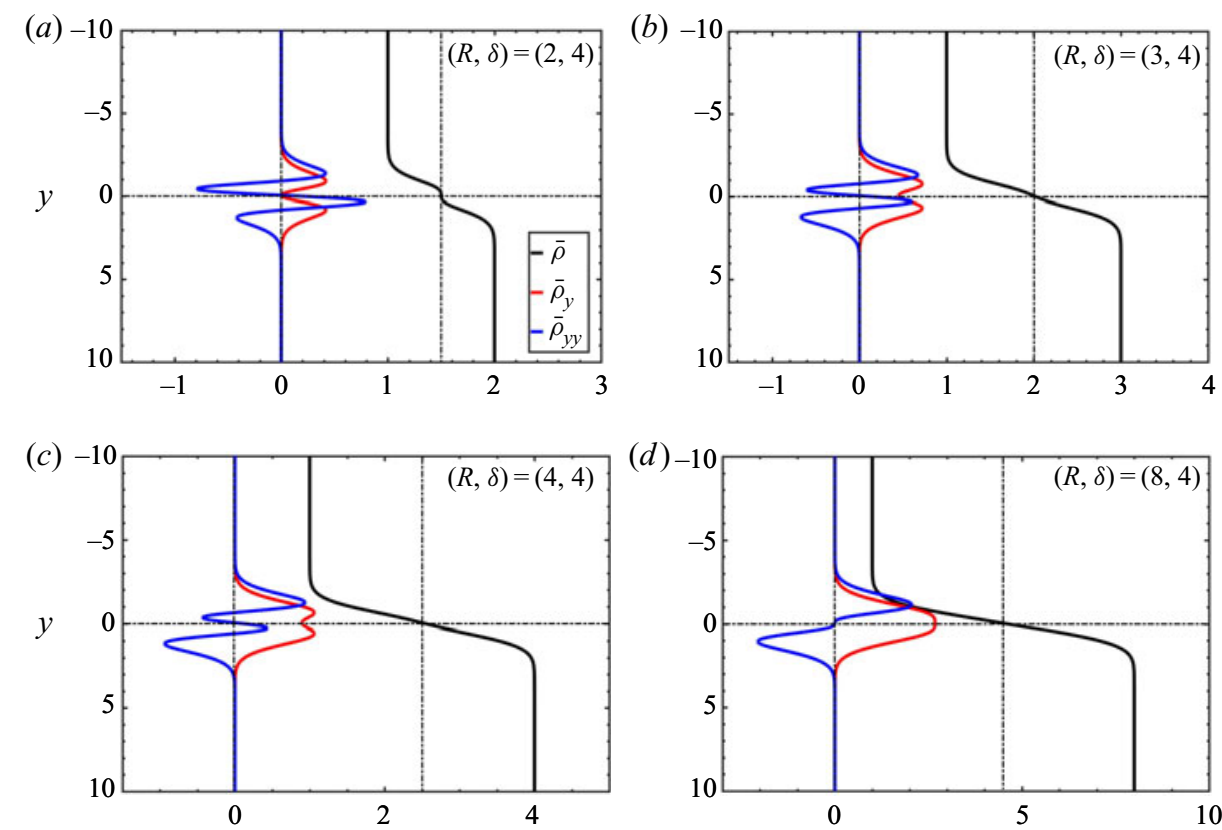

Figure 6. Typical base-state density profiles and their spatial derivatives in the DDD regime $\left(R^{2 / 3} \leq \delta<R^{2}\right)$.

Hence, an adverse dynamic density jump $\Delta \rho_{m}$ cannot be defined. The necessary condition for such base flows to be unstable is the presence of a point on either side of the initial interface where the second derivative of the base-state density profile is 0 , i.e. it changes its sign on either side of the initial interface (Gopalakrishnan 2020). In these flows, the instability arises as stationary modes unlike in the other unstable regimes, where the growth rates can be real or complex.

\section{Scalings in the RT-DLC regime $(R<1, \delta<1)$ and pure DLC regime $(R>1, \delta<1)$}

The flow dynamics can also be influenced by DLC modes if $\delta<1$. We analyse here the scalings of the mixing velocity $U$ in this case, both in the RT regime $(R<1)$ and in the pure DLC regime $(R>1)$. When $R^{2} \leq \delta<1$, the base-state density profiles are 

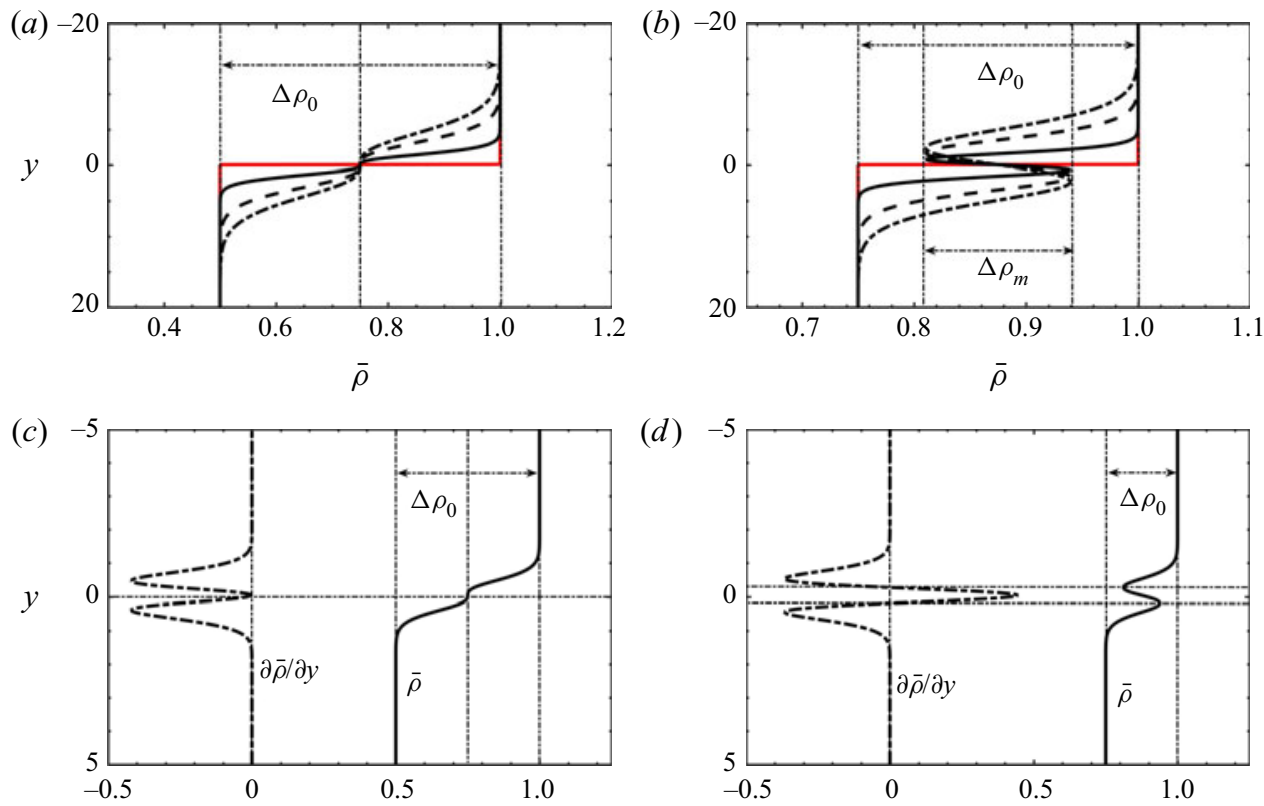

Figure 7. Base-state density profiles in RT regimes with $(a) R^{2} \leq \delta<1$ and $(b) R_{c} \leq \delta<R^{2}$ where the unstable initial density difference $\Delta \rho_{0}$ (shown in red) is larger than the stable stratification $\Delta \rho_{m}$ that develops at $t>0$. $(c, d)$ The base-state density profile and its spatial derivative $\partial \bar{\rho} / \partial y$. Parameter settings: $(a, c)$ $\delta=0.25, R=0.50, \Delta \rho_{m}=0, \Delta \rho_{0}=0.50 ;(b, d) \delta=0.25, R=0.75, \Delta \rho_{m}=-0.19, \Delta \rho_{0}=0.25$.

monotonic (see figure $7 a, c$ ) with the unstable initial density difference, $\Delta \rho_{0}=1-R$, driving the flow dynamics as observed in single-species RT flows. If $R_{c} \leq \delta<R^{2}$, the faster diffusion of A downwards results in a non-monotonic base-state density profile once $t>0$. A buoyantly stable zone where $\partial \bar{\rho} / \partial y>0$ develops then across the initial interface (figure $7 b, d$ ). The amplitude of the density difference across that stable layer is the dynamic density jump $\Delta \rho_{m}$ given by $(4.1) ; \Delta \rho_{m}$ between the minimum and maximum in density has here a negative value as, locally, the density stratification is stable around the interface.

We can then distinguish two different zones in the region of the parameter space $\delta<R^{2}$. They are separated by the curve $\delta=R_{c}$ given by $\delta=R^{n}$ (where $n \approx 6.8$ ) (Carballido-Landeira et al. 2013) at which $\Delta \rho_{m}=\Delta \rho_{0}$. First, if $R_{c} \leq \delta<R^{2}<1$, we have $\Delta \rho_{0}>\left|\Delta \rho_{m}\right|$ (see figure $7 b, d$ ) and the growth of the RT mixing zone is controlled by $\Delta \rho_{0}$. Once $\delta<R_{c}$, we have $\Delta \rho_{0}<\left|\Delta \rho_{m}\right|$ as the two extrema of the non-monotonic density profile lie outside the range of initial values of density (figure $8 a$ ). A local buoyantly stable layer separates the solutions across the initial interface while zones where locally a denser solution overlies a less dense one develop on either side of the initial interface. The typical base-state density profile and its spatial derivative is illustrated in figure $8(c)$. An adverse density jump $\Delta \rho_{m}^{\prime}$ can be defined across the zone where locally $\partial \bar{\rho} / \partial y<0$ on either side of the initial interface (figure $8 a$ ). Its value can be computed as

$$
\Delta \rho_{m}^{\prime}=\frac{\Delta \rho_{0}-\Delta \rho_{m}}{2}, \quad \text { for } \Delta \rho_{0}<\left|\Delta \rho_{m}\right|,
$$

with $\Delta \rho_{m}$ given by (4.1).

Similar features i.e. a non-monotonic density profile such that $\left|\Delta \rho_{0}\right|<\left|\Delta \rho_{m}\right|$ but with both $\Delta \rho_{0}<0$ and $\Delta \rho_{m}<0$ can be observed in pure DLC regimes when $R \geq 1, \delta<1$, 
(a)

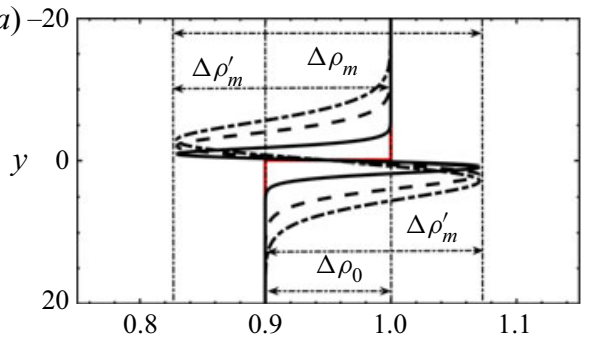

(c)

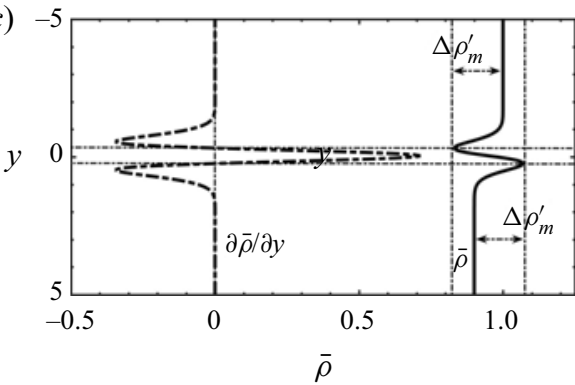

(b)

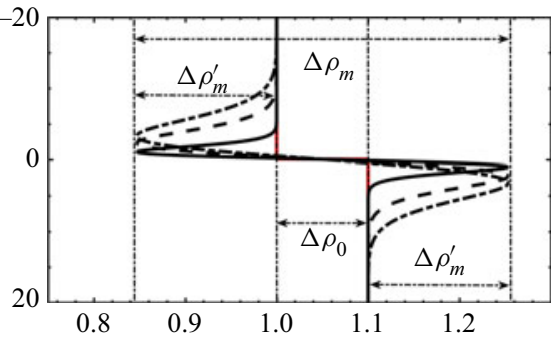

(d)

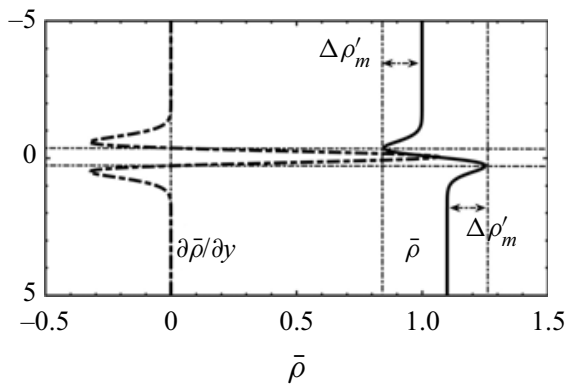

Figure 8. Base-state density profiles in $(a)$ the RT-DLC mixed-mode regime and $(b)$ in the pure DLC regime. $(c, d)$ Corresponding spatial derivatives and the adverse density jump $\Delta \rho_{m}^{\prime}$. Parameter settings: $(a, c) \delta=0.25$, $R=0.90, \Delta \rho_{0}=0.10, \Delta \rho_{m}=-0.24, \Delta \rho_{m}^{\prime}=0.17 ;(b, d) \delta=0.25, R=1.10, \Delta \rho_{0}=-0.10, \Delta \rho_{m}=-0.41$, $\Delta \rho_{m}^{\prime}=0.15$.

as shown in figure $8(b, d)$. As $R \geq 1$, the initial density difference $\Delta \rho_{0}$ is negative, corresponding to an initially buoyantly stable configuration. In these flows, as discussed in $\S 3$, the system destabilises due to the differential diffusion of the solutes which induces an adverse density stratification on either side of the initial interface, similar to the RT-DLC mixed-mode regime. This adverse density jump $\Delta \rho_{m}^{\prime}$, given by (6.1), is the density difference across the regions where $\partial \bar{\rho} / \partial y<0$ as shown in figure $8(d)$.

The flow dynamics corresponding to the various base-state density profiles for $\delta<1$ shown in figures 7 and 8 is summarised in figure 9 giving the spatial distribution of the density field at a given time during the nonlinear regime. In the RT regime, fingers extend all across the initial position of the interface (figure $9 a, b$ ) with some fingers showing a Y-shape if $R_{c} \leq \delta<R^{2}$ (figure $9 b$ ). The corresponding density variation along the black and red lines is shown in figure $9(e, f)$. When entering the RT-DLC mixed-mode regime $\left(\delta<R_{c}\right)$, DLC features, such as local deformation of the tip of the fingers into Y-shaped antennae, appear in addition to the RT modulation of the interface (Carballido-Landeira et al. 2013). Figure 9(c) shows an example of such a RT-DLC mixed mode. Figure $9(d)$ illustrates the pure DLC dynamics characterised by a stable middle interface and convective zones developing on either side of it. The origin of the DLC mechanism can be noted in the density map where a low density zone is seen above the interface while an accumulation zone with a larger density develops below the interface. As can be seen in figure $9(h)$ the density difference driving the convection of the fingers is $\approx 0.16$ both above and below the interface which corresponds to the value of $\Delta \rho_{m}^{\prime}=0.16$.

Figure 10(a) plots the averaged mixing length for the RT-DLC regime. The mixing length is here still measured as the distance between the uppermost tip of fingers in the top layer to the lowest tip of the fingers in the lower layer. Figure 10(b) shows that, as $R$ increases, the mixing velocity $U$ decreases. This is due to the fact that the driving density jump decreases as well. We find numerically that the mixing velocity $U$ scales throughout 
S.S. Gopalakrishnan, B. Knaepen and A. De Wit

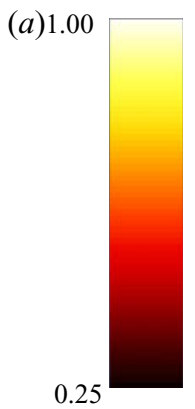

$(b) 1.00$

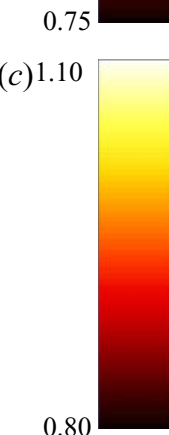

(d) 1.30

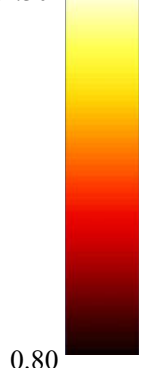

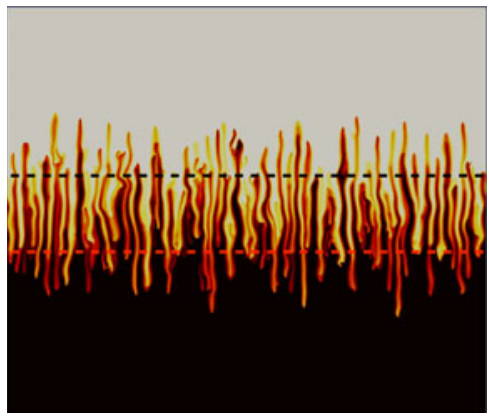
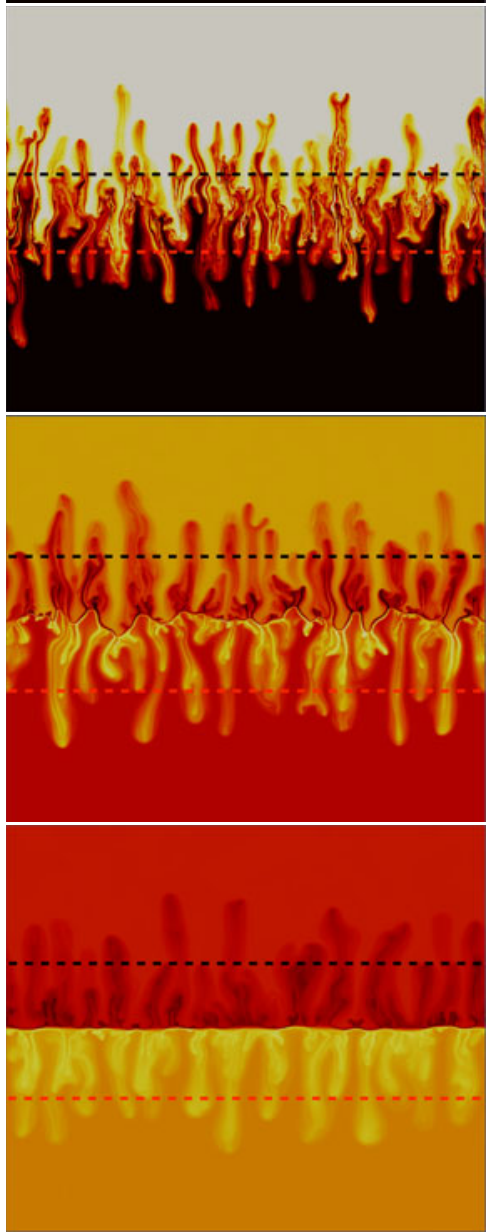

(e)

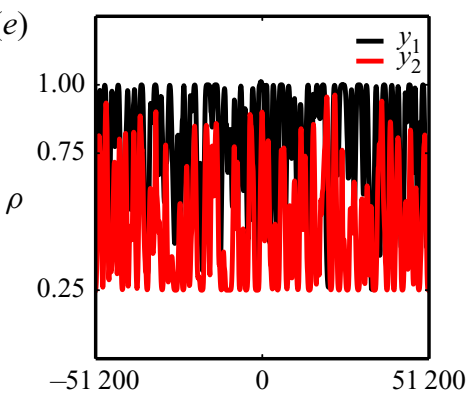

$(f)$

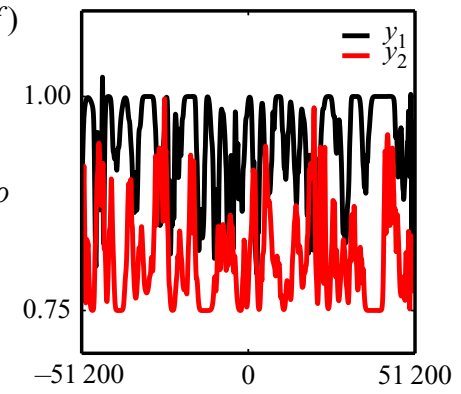

(g)

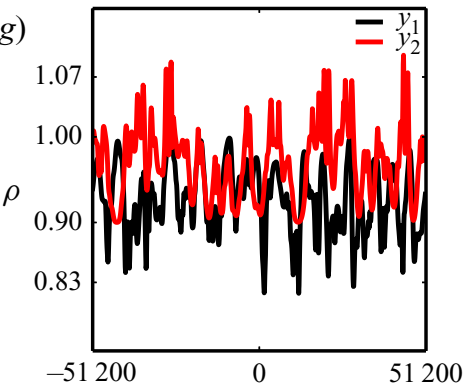

(h)

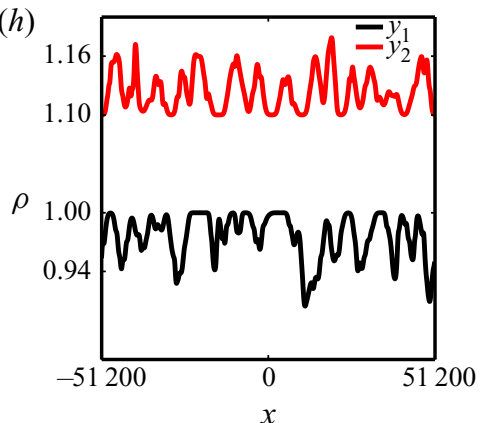

Figure 9. Spatial distribution of density fields illustrating the transition from flows dominated by $(a, b)$ RT effects, to the $(c)$ RT-DLC mixed-mode scenario and eventually to the $(d)$ pure DLC mechanism. The variation of density for the corresponding flow fields along the black and red lines are shown in $(e, f, g, h)$. Parameter settings: $\delta=0.25$ and (a) $R=0.25$, (b) $R=0.75,(c) R=0.90,(d) R=1.1$.

the $\delta<1$ zone as $U \sim 0.8 \Delta \rho_{m}^{*}$ where $\Delta \rho_{m}^{*}$ is defined as

$$
\begin{gathered}
\Delta \rho_{m}^{*}=\Delta \rho_{0}, \quad \text { for }\left|\Delta \rho_{0}\right| \geq\left|\Delta \rho_{m}\right|, \\
\Delta \rho_{m}^{*}=0.5 \Delta \rho_{m}^{\prime}, \quad \text { for }\left|\Delta \rho_{0}\right|<\left|\Delta \rho_{m}\right| .
\end{gathered}
$$


(a)

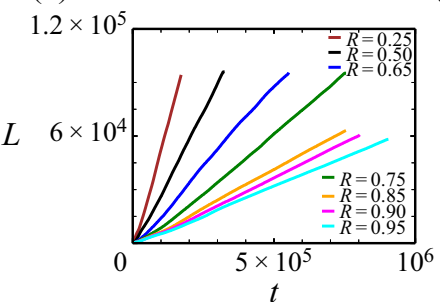

(d)

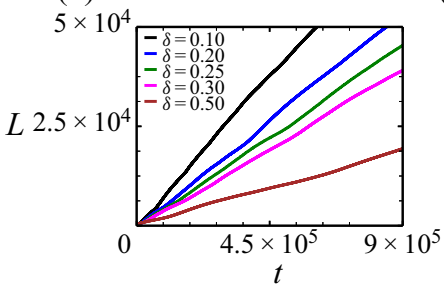

(b)

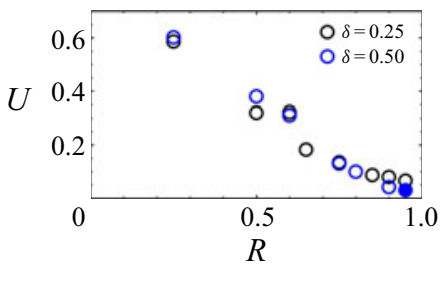

(e)

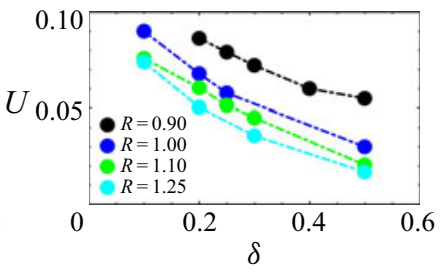

(c)

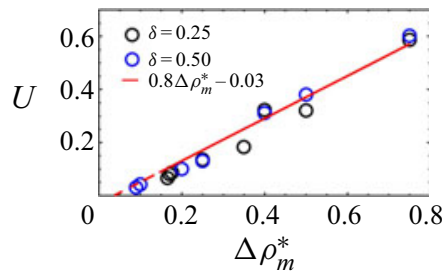

$(f)$

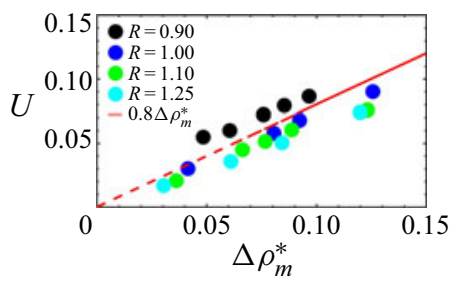

Figure 10. Temporal evolution of the mixing length $L$ (averaged over 20 simulations) for $(a)$ variable $R$ at $\delta=0.25$ and $(d)$ variable $\delta$ at $R=1.1$. Mixing velocity $U$ as a function of $(b) R$ and $(e) \delta$, and its variation with the adverse density jump $\Delta \rho_{m}^{*}$ in $(c, f)$. Open circles indicate when $\Delta \rho_{m}^{*}$ is given by the initial density difference $\Delta \rho_{0}$, and filled circles by (6.3). The maximum deviation in the values of $U$ arising from the seeded initial conditions over 20 simulations is of the order $10^{-3}$.

The scaling of the mixing velocity $U$ as a function of the adverse density jump $\Delta \rho_{m}^{*}$ is shown in figure $10(c)$. In figure $10(b, c, e, f)$, the open circles indicate that the driving force comes from the unstable initial stratification $\Delta \rho_{0}$ for $\delta>R_{c}$ and filled circles are driven by $\Delta \rho_{m}^{*}=0.5 \Delta \rho_{m}^{\prime}$ when $\delta<R_{c}$. As in the earlier flow regimes, we observe a linear relationship between $U$ and the adverse density stratification $\Delta \rho_{m}^{*}$, with $U=0.8 \Delta \rho_{0}$ for $R_{c} \leq \delta$, and $U=0.8 \Delta \rho_{m}^{*}=0.4 \Delta \rho_{m}^{\prime}$ for $\delta<R_{c}$.

The evolution of the average mixing length in the DLC regime is shown in figure $10(d)$ for different values of $\delta$ and $R=1.1$. As $\delta$ decreases at a fixed value of $R$, the rate of diffusion of the solute from the upper solution to the lower one increases, which results in an increased value of the adverse density jump $\Delta \rho_{m}^{\prime}$ and thus to an increased mixing velocity $U$ (figure $10 e$ ). The mixing velocity $U$ as a function of the adverse density jump $\Delta \rho_{m}^{*}$ given by (6.2) and (6.3) is plotted in figure $10(f)$ where a linear scaling, $U=0.8 \Delta \rho_{m}^{*}$, can be observed. The same scaling law applies thus to both the RT-DLC mixed-mode regime and pure DLC flows, similarly to the RT-DD and DD flows. The fact that $\Delta \rho_{m}^{*}=0.5 \Delta \rho_{m}^{\prime}$ when $\left|\Delta \rho_{m}\right|>\Delta \rho_{0}$ is due to the fact that the density jump $\Delta \rho_{m}^{\prime}$ can develop only towards one side as it is bounded on the other side by a stable barrier. The extent of this stable barrier is given by $\Delta \rho_{m}$ (4.1).

The transition of the adverse density jump from $\Delta \rho_{0}$ to $\Delta \rho_{m}^{*}=0.5 \Delta \rho_{m}^{\prime}$ in the $(R, \delta)$ plane when $\delta<1$ is summarised in figure 11(a). Figure 11(b) shows isocontours of the adverse density jump in the $\delta<1$ zone. The curve $\delta=R_{c}$, given by $\delta=R^{n}(n=6.8)$ shown using a dotted line demarcates the switch from the RT regime to the RT-DLC mixed-mode and pure DLC regimes. For $\delta>R_{c}, \Delta \rho_{0}=1-R$ is at play. When $\delta<R_{c}$, $\Delta \rho_{m}^{*}=0.5 \Delta \rho_{m}^{\prime}$ fixes the dynamics. The value of $\Delta \rho_{m}^{\prime}$ is given by (6.1) and decreases with an increase in $\delta$ at a fixed $R$. For small values of $\delta(<0.2), \Delta \rho_{m}^{\prime}$ stays roughly constant as a function of $R$, whereas at higher values of $\delta$ it decreases slowly. Figure 11(c) summarises the scaling $U=0.8 \Delta \rho_{m}^{*}$ across values dominated by $\Delta \rho_{0}$ (in black) and those ruled by $\Delta \rho_{m}^{\prime}$ (in blue). 

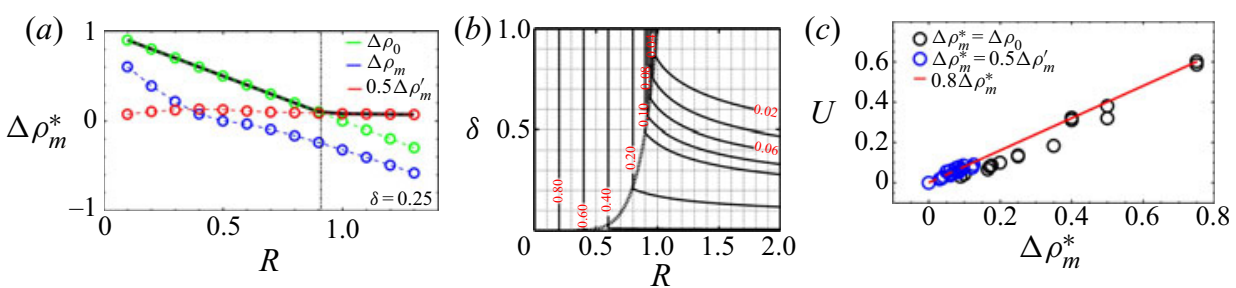

Figure 11. (a) Variation of $\Delta \rho_{0}, \Delta \rho_{m}$ and $0.5 \Delta \rho_{m}^{\prime}$ as a function of $R$ for $\delta=0.25$, with $\Delta \rho_{m}^{*}$ denoted by the thick black line. The switch of $\Delta \rho_{m}^{*}$ from $\Delta \rho_{0}$ to $0.5 \Delta \rho_{m}^{\prime}$ occurs at $R=0.915$. (b) Contours of the adverse density jump $\Delta \rho_{m}^{\prime}$ which governs the scaling of $U$ in the $(R, \delta)$ plane for $\delta<1$ illustrating the transition from the RT dominant zone for $R<R_{c}$ shown by the thick dotted line to the DLC-dominated regime when $R>R_{C}$. In the RT zone, the adverse density jump is $\Delta \rho_{0}=1-R$ and the scaling of the mixing velocity is $U=0.8 \Delta \rho_{0}$. In the RT-DLC mixed-mode and pure DLC regimes, we have $U=0.8 \Delta \rho_{m}^{*}=0.5 \Delta \rho_{m}^{\prime}$. This is shown in panel (c), where the velocity $U$ is shown as a function of $\Delta \rho_{m}^{*}$ for $\delta<1(\delta=[0.10,0.20,0.25,0.30,0.50])$, with $R$ ranging from 0.25 to 1.25 .

\section{Summary and discussion}

Different buoyantly unstable scenarios can be obtained depending on the values of the two non-dimensional parameters $R$ and $\delta$ that govern the flow dynamics. The miscible interface is subject to a RT instability when $R<1$. The single-species RT instability occurs when $\delta=1$. In that case, the density is decreasing monotonically along the gravity field and $U$ scales as $0.8 \Delta \rho_{0}$ where $\Delta \rho_{0}=1-R$ is the initial density difference.

In the case of two-species RT-DD stratification, if the species in the lower solution diffuses faster $(\delta>1)$, the density profiles have a non-monotonic spatial dependence with a maximum in density above the interface and a minimum in density in the lower layer as soon as $t>0$. The adverse density jump $\Delta \rho_{m}$ between these extrema is larger than the initial density difference $\Delta \rho_{0}$, and can be computed analytically from the diffusive base-state concentration profiles using the values of $R$ and $\delta$ via (4.1). The mixing velocities computed in the corresponding nonlinear RT regime are found to scale linearly as $U=0.8 \Delta \rho_{m}$. This is due to the fact that the density difference experienced by the fingers with the solution ahead of them during their nonlinear evolution is approximately $\Delta \rho_{m}$. For $\delta>1$ and a buoyantly stable initial configuration $(R \geq 1)$ such that $\Delta \rho_{0}<0$, DD effects result in an adverse density difference $\Delta \rho_{m}>0$ across the miscible interface due to the faster upwards diffusion of the species from the lower layer. In the nonlinear regime, the mixing velocity $U$ is found to scale linearly with this adverse density difference as $U=0.8 \Delta \rho_{m}$. The footprint of $\Delta \rho_{m}$ can be observed in the nonlinear regime from the spatial distribution of the density fields with the density difference between the fingers and their surroundings being $\Delta \rho_{m}$.

If $\delta<1$, other scenarios are encountered. If $R^{2} \leq \delta<1$, the RT density profiles are monotonically decreasing downwards and the flow dynamics is governed by the initial density difference $\Delta \rho_{0}$ with $U=0.8 \Delta \rho_{0}$. For $R_{c} \leq \delta<R^{2}$, the non-monotonic density profiles feature a locally stable stratification across the initial interface where $\Delta \rho_{m}<0$. In this regime, the initial density difference $\Delta \rho_{0}$ still drives the convective mixing globally and $U=0.8 \Delta \rho_{0}$ even though the influence of differential diffusion can be seen at the tips of some fingers. For $\delta \leq R_{c}<1$, when the absolute value of the locally stable stratification $\Delta \rho_{m}(<0)$ exceeds the initial density difference $\Delta \rho_{0}>0$, an adverse density difference $\Delta \rho_{m}^{\prime}$ given by (6.1) can be observed on either side of the interface. In this RT-DLC mixed-mode regime, where DLC effects strongly influence the RT flow dynamics, 'Y-shaped antenna-like' structures can be observed developing around 


\section{Scaling of buoyancy-driven fingers in porous media}

the interface. Similar features in the density profiles can also be observed in a buoyantly stable stratification $R>1$ with $\delta<1$ where only pure DLC mechanisms are present. The mixing velocities in these regimes decrease with increasing $R$ and $\delta$, and are found to scale linearly as $U=0.8 \Delta \rho_{m}^{*}=0.4 \Delta \rho_{m}^{\prime}$. For the RT-DLC mixed-mode and pure DLC scenarios, the instability develops separately above and below the interface, with $\Delta \rho_{m}^{*}$ driving the convection that develops independently on either side of the mixing zone.

In all the scenarios discussed in this study, the most striking observation is that the driving force for the mixing velocity of the fingers is always the density difference experienced by the fingers with their surroundings. This density difference, although computed analytically from the base-state diffusive density profiles, persists in the nonlinear regime, and imprints therefore its influence on the mixing velocity $U$. This is illustrated in figure 12 which shows the distribution of the density field within our computational domain at a given time in the nonlinear regime for the different scenarios discussed in the present study in the $(R, \delta)$ parameter space. For $(a) \mathrm{RT}$ and $(e)$ DD instabilities, the distribution of the density field shows that the density difference experienced by the fingers with their surroundings on either side of the initial interface is $\Delta \rho_{m}$. When $R_{c} \leq \delta<1$ i.e. for RT-dominated flows $(b, c)$, the initial density difference $\Delta \rho_{0}=1-R$ drives the convective mixing. For the flows corresponding to the $(d)$ RT-DLC mixed-mode regime and the $(f)$ pure DLC, $\Delta \rho_{m}^{*}=0.5 \Delta \rho_{m}^{\prime}$ is the density difference which governs the evolution of the mixing length.

A summary of the different scalings can be seen in the various shaded zones of figure 1 with the shaded blue region corresponding to $U=0.8 \Delta \rho_{m}$ (RT-DD regime and pure DD modes), the green region to $U=0.8 \Delta \rho_{0}$ (RT dominant modes at $\delta<1$ ) and the pink region to $U=0.8 \Delta \rho_{m}^{*}=0.4 \Delta \rho_{m}^{\prime}$ (RT-DLC mixed mode and pure DLC modes).

The fact that the fingers extend vertically at a speed proportional to the largest adverse density jump may sound intuitive at first sight. It certainly is intuitive in the case of the pure one-species RT instability where the density difference $\Delta \rho_{0}$ between the upper solution of A and the lower layer of pure solvent controls the dynamics. The situation is less intuitive for two-species stratification. If you consider solutions of salt and sugar, different velocities will be observed even if starting from the same $\Delta \rho_{0}$ because salt diffuses approximately 3 times faster than sugar. If the denser layer of salt overlies a less dense solution of sucrose, we have $\delta=1 / 3$. Then, even if starting with the same $\Delta \rho_{0}$, the RT instability will be influenced by DLC effects for $R_{c} \leq R<1$ in the RT-DLC zone. The mixing velocity will then scale linearly with $\Delta \rho_{m}^{*}$. If, in contrast, we start from a denser layer of sugar above a less dense solution of salt then $\delta=3$ and $\Delta \rho_{m}$ becomes the relevant density jump. Table 2 from the supplemental information of Gopalakrishnan et al. (2018) lists a series of values of $\Delta \rho_{0}$ and $\Delta \rho_{m}$ that can guide experimentalists in new experiments devoted to test our predictions.

Our results also allow us to understand why, in the experiments of Pringle and Glass (Pringle \& Glass 2002) on the DD instability, the velocity with which the fingers advance vertically remains almost the same even when concentrations are varied at a fixed $\delta$ (see figure 10 in their article). This can be explained by the fact that the change in concentrations was done by maintaining $R$ at a constant value as they were changing the values of initial concentrations $A_{0}$ and $B_{0}$ in the same proportion (see table 1 of their manuscript).

\section{Conclusions}

A miscible horizontal interface separating solutions of two different species can deform into finger-like structures in the gravity field due to buoyancy-driven instabilities like the 

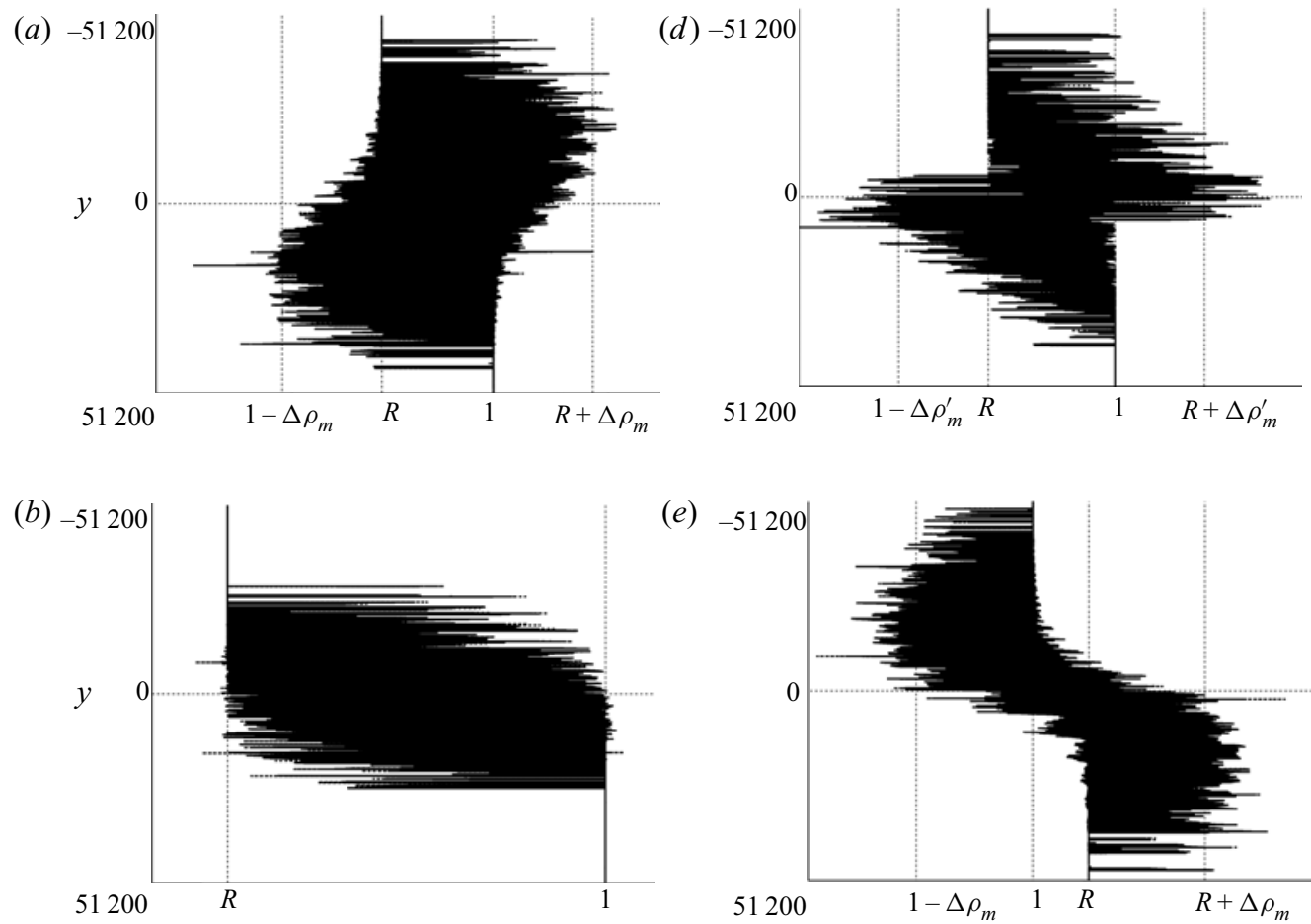

(e)

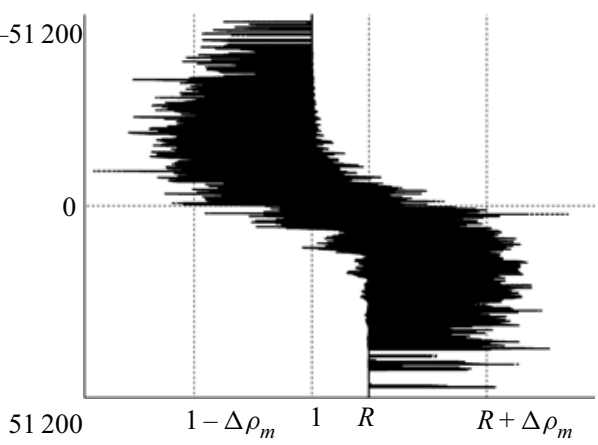

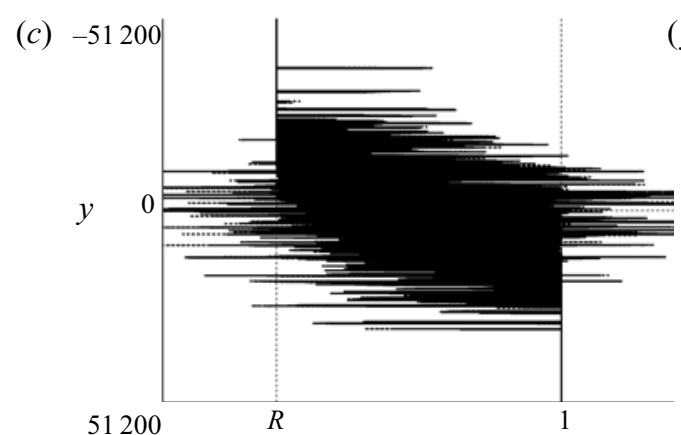

$\operatorname{Dist}(\rho)$

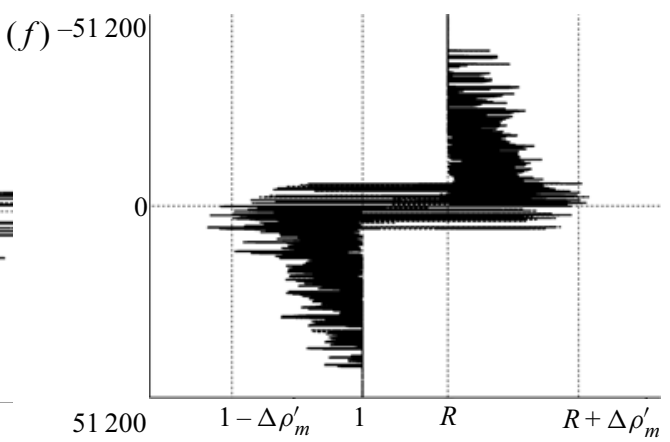

$\operatorname{Dist}(\rho)$

Figure 12. Distribution of the values of the density field within our computational domain at a given time in the nonlinear regime for the various scenarios discussed in the present study. The density difference $\Delta \rho$ driving the evolution of the mixing length on either side of the initial interface $y=0$ is: (a) RT-DD: $R<1, \delta \geq 1$, $\Delta \rho=\Delta \rho_{m} ;$; (b) RT: $R<1, R^{2}<\delta<1, \Delta \rho=\Delta \rho_{0}=1-R ;$ (c) RT: $R<1, R_{c}<\delta<R^{2}, \Delta \rho=\Delta \rho_{0}$; (d) RT-DLC: $R<1, \delta<R_{c}, \Delta \rho=0.5 \Delta \rho_{m}^{\prime}$; (e) DD: $R \geq 1, \delta>1, \Delta \rho=\Delta \rho_{m}$; (f) DLC: $R \geq 1, \delta<1$, $\Delta \rho=0.5 \Delta \rho_{m}^{\prime}$. Parameter settings: $(a) R=0.868, \delta=2,(b) R=0.25, \delta=0.25,(c) R=0.75, \delta=0.25,(d)$ $R=0.90, \delta=0.25,(e) R=1.1, \delta=3,(f) R=1.1, \delta=0.25$.

RT, DD and DLC instabilities. We have here quantified in each regime the influence of differential diffusion between the two species on the mixing velocity in the nonlinear regime when the flow is solution of Darcy's law. These differential diffusion effects can induce non-monotonic base-state density profiles in which an adverse density difference $\left(\Delta \rho_{m}\right.$ or $\Delta \rho_{m}^{*}$ ) can develop as soon as the two fluids are in contact and start to mix by diffusion. We have computed numerically the mixing velocity $U$ of the fingers as the 
slope of the temporal evolution of the averaged mixing length in the nonlinear regime. We show that, throughout the parameter space $(R, \delta), U$ scales linearly with the largest adverse density difference present in the density profile. This adverse density jump $\left(\Delta \rho_{m}\right.$ if $\delta>1$ and $\Delta \rho_{m}^{*}$ if $\delta<1$ ) can be different from the initial density jump $\Delta \rho_{0}$ thanks to differential diffusion. It can be computed analytically as a function of $R$ and $\delta$. This result demonstrates that the mixing properties can be predicted in two-species stratifications knowing solely the initial concentrations, the solutal expansion coefficients and the diffusion coefficients of the various species involved, which fix the values of the buoyancy ratio $R$ and of the diffusivity ratio $\delta$. This paves the way to a control of the mixing of two miscible layers by a careful choice of the solutes dissolved in these layers. Their nature and the order in which they are layered fixes the ratio of diffusivities $\delta$ and hence the accessible double-diffusive effects (DD if $\delta>1$ or DLC if $\delta<1$ ). A subsequent choice of their initial concentrations fixes then the buoyancy ratio $R$ and thus the dynamic density jump controlling the growth of the mixing length of the buoyancy-driven fingers. This control strategy, illustrated here for differential diffusion effects, can easily be adapted to other processes (like non-ideal mixing or chemical reactions for instance) that are able to induce non-monotonic density profiles (De Wit 2020) or to multispecies stratifications. Moreover, as the control depends only on local adverse density jumps, which are obtained analytically from the diffusion equation and not from the flow equations, it will be interesting to test if the scalings demonstrated here using porous medium flows have equivalent robust scalings for other flow equations as well.

Acknowledgements. We thank the anonymous referees for suggesting several ways to improve the quality and presentation of the article.

Funding. A.D. acknowledges the financial support of FRS-FNRS under the CONTROL research programme.

Declaration of interest. The authors report no conflict of interest.

Author ORCIDs.

(1) S.S. Gopalakrishnan https://orcid.org/0000-0002-8283-3983;

(1) B. Knaepen https://orcid.org/0000-0002-2248-4558;

(1) A. De Wit https://orcid.org/0000-0002-3231-0906.

\section{REFERENCES}

Almarcha, C., Trevelyan, P.M.J., Grosfils, P. \& De Wit, A. 2010 Chemically driven hydrodynamic instabilities. Phys. Rev. Lett. 104, 044501.

BATCHELOR, G.K. 1954 Heat convection and buoyancy effects in fluids. Q. J. Roy. Meteor. Soc. 80, 339-356.

BatcheloR, G.K. 1967 An Introduction to Fluid Dynamics. Cambridge University Press.

Carballido-Landeira, J., Trevelyan, P.M.J., Almarcha, C. \& De Wit, A. 2013 Mixed-mode instability of a miscible interface due to coupling between Rayleigh-Taylor and double-diffusive convective modes. Phys. Fluids 25, 024107.

COOPER, C.A., Glass, R.J. \& TYLER, S.W. 1997 Experimental investigation of the stability boundary for double-diffusive finger convection in a Hele-Shaw cell. Water Resour. Res. 33, 517-526.

DAVIES WyKeS, M.S. \& DAlZiel, S.B. 2014 Efficient mixing in stratified flows: experimental study of Rayleigh-Taylor unstable interfaces within an otherwise stable stratification. J. Fluid Mech. 756, 1027-1057.

De PaOli, M., Zonta, F. \& Soldati, A. 2019 Rayleigh-Taylor convective dissolution in confined porous media. Phys. Rev. Fluids 4, 023502.

DE Wit, A. 2016 Chemo-hydrodynamic patterns in porous media. Phil. Trans. R. Soc. A 374, 20150419.

DE Wit, A. 2020 Chemo-hydrodynamic patterns and instabilities. Annu. Rev. Fluid Mech. 52, 531-555.

Donev, A., Nonaka, A., Bhattacharjee, A.K., Garcia, A.L. \& Bell, J.B. 2015 Low Mach number fluctuating hydrodynamics of multispecies liquid mixtures. Phys. Fluids 27, 037103.

Dullien, F.A. 2012 Porous Media: Fluid Transport and Pore Structure. Academic Press. 


\section{S.S. Gopalakrishnan, B. Knaepen and A. De Wit}

Fernandez, J., Kurowski, P., Limat, L. \& Petitjeans, P. 2001 Wavelength selection of fingering instability inside Hele-Shaw cells. Phys. Fluids 13, 3120-3125.

Fernandez, J., Kurowski, P., Petitjeans, P. \& Meiburg, E. 2002 Density-driven unstable flows of miscible fluids in a Hele-Shaw cell. J. Fluid Mech. 451, 239-260.

GANDhi, J. \& TREVElyan, P.M.J. 2014 Onset conditions for a Rayleigh-Taylor instability with step function density profiles. J. Engng Maths 86, 31-48.

Gopalakrishnan, S.S. 2020 On the instability of buoyancy-driven flows in porous media. J. Fluid Mech. 892, A13.

Gopalakrishnan, S.S., Carballido-landeira, J., De Wit, A. \& Knaepen, B. 2017 Relative role of convective and diffusive mixing in the miscible Rayleigh-Taylor instability in porous media. Phys. Rev. Fluids 2, 012501.

Gopalakrishnan, S.S., Carballido-Landeira, J., Knaepen, B. \& De Wit, A. 2018 Control of Rayleigh-Taylor instability onset time and convective velocity by differential diffusion effects. Phys. Rev. E 98, 011101(R).

GrEEN, T. 1984 Scales for double-diffusive fingering in porous media. Water Resour. Res. 20, 1225-1229.

GRIFFITHS, R.W. 1981 Layered double-diffusive convection in porous media. J. Fluid Mech. 102, 221-248.

HiLl, S. 1952 Channelling in packed columns. Chem. Engng Sci. 1, 247-253.

Huppert, H.E. \& Neufeld, J.A. 2014 The fluid mechanics of carbon dioxide sequestration. Annu. Rev. Fluid Mech. 46, 255-72.

Huppert, H.E. \& SPARKS, R.S.J. 1984 Double-diffusive convection due to crystallization in magmas. Annu. Rev. Fluid Mech. 12, 11-37.

Huppert, H.E. \& Turner, J.S. 1981 Double-diffusive convection. J. Fluid Mech. 106, 299-329.

Jenny, P., LEe, J.S., MeYer, D.W. \& TChelepi, H.A. 2014 Scale analysis of miscible density-driven convection in porous media. J. Fluid Mech. 749, 519-541.

LAWrie, A.G.W. \& DAlzIEL, S.B. 2011 Turbulent diffusion in tall tubes. II. Confinement by stratification. Phys. Fluids 23, 085110.

Lemaigre, L., Budroni, M.A., Riolfo, L.A., Grosfils, P. \& De Wit, A. 2013 Asymmetric Rayleigh-Taylor and double-diffusive fingers in reactive systems. Phys. Fluids 25, 014103.

MANICKAM, O. \& HoMSY, G.M. 1995 Fingering instabilities in vertical miscible displacement flows in porous media. J. Fluid Mech. 288, 75-102.

Martin, J., Rakotomalala, N. \& Salin, D. 2002 Gravitational instability of miscible fluids in a Hele-Shaw cell. Phys. Fluids 14, 902-905.

Menand, T. \& Woods, A.W. 2005 Dispersion, scale, and time dependence of mixing zones under gravitationally stable and unstable displacements in porous media. Water Resour. Res. 41, W05014.

Moureau, V., Domingo, P. \& Vervisch, L. 2011 Design of a massively parallel CFD code for complex geometries. C. R. Méc 339, 141-148.

NakAnishi, Y., Hyodo, A., WAng, L. \& Suekane, T. 2016 Experimental study of 3D Rayleigh-Taylor convection between miscible fluids in a porous medium. Adv. Water Resour. 97, 224-232.

Pringle, S.E. \& GLASS, R.J. 2002 Double-diffusive finger convection: influence of concentration at fixed buoyancy ratio. J. Fluid Mech. 462, 161-183.

RADKo, T. 2013 Double Diffusive Convection. Cambridge University Press.

Schmitt, R.W. 1994 Double diffusion in oceanography. Annu. Rev. Fluid Mech. 26, 255-285.

Schmitt, R.W., Ledwell, J.R., Montgomery, E.T., Polzin, K.L. \& Toole, J.M. 2005 Enhanced diapycnal mixing by salt fingers in the thermocline of the tropical Atlantic. Science 308, 685.

Scott, D.R. \& Stevenson, D.J. 1986 Magma ascent by porous flow. J. Geophys. Res. 91, 9283-9296.

Slim, A.C. 2014 Solutal-convection regimes in a two-dimensional porous medium. J. Fluid Mech. 741, 461-491.

Stamp, A.P., Hugher, G.O., Nokes, R.I. \& Griffiths, R.W. 1998 The coupling of waves and convection. J. Fluid Mech. 372, 231-271.

Teng, Y., JiAng, L., FAn, Y., Liu, Y., Wang, D., Abudula, A. \& Song, Y. 2017 Quantifying the dynamic density driven convection in high permeability packed beds. Magn. Reson. Imag. 39, 168-174.

Trevelyan, P.M.J., Almarcha, C. \& DE Wit, A. 2011 Buoyancy-driven instabilities of miscible two-layer stratifications in porous media and Hele-Shaw cells. J. Fluid Mech. 670, 38-65.

TURner, J.S. 1979 Buoyancy Effects in Fluids. Cambridge University Press.

TURnER, J.S. \& STOMMEL, H. 1964 A new case of convection in the presence of combined vertical salinity and temperature gradients. Proc. Natl Acad. Sci. USA 52, 49-53.

Wooding, R.A. 1969 Growth of fingers at an unstable diffusing interface in a porous medium or Hele-Shaw cell. J. Fluid Mech. 39, 477-495. 